\title{
Optical Camouflage Effect Objective Evaluation Method Research Under The Condition of Complex Backgrounds
}

\author{
Jianfei Qin ${ }^{\text {a }}$, Liyong Qu , Liyan Zhu , Jianghua Hu Shuwei Song \\ National Key Laboratory of Electromagnetic Environment Effects and Electro-optical Engineering, PLA University of Science and \\ Technology, Nanjing 210007, China ${ }^{5} 94860$ PLA Troops, Nanjing 210007, China
}

\begin{abstract}
According to the problem that the intelligence is not high of the soccer robot, using the mechanical theory as a guide, making some mechanical analyses and calculations on the pressure and transmutation states of chip kick mechanics, and conducting optimal design too, then making the structure of chip kick mechanics more and more rationalization. Experiments show that the new soccer robot controller features a quick response and high servo rigidity, and provide a kind of method for improving and perfecting the soccer robot control system, at the same time,filling the needs of producing
\end{abstract}

\section{Introduction}

Objective evaluation of camouflage effect of camouflage technology has important direction, the camouflage effect evaluation models are mainly texture analysis[1,2], color histogram method [3], spectral reflection characteristics method [4], etc. Texture analysis makes the objective and accurate evaluation of texture is difficult due to texture indistinct and difficult to measure. The method of gray co-occurrence matrix can't show a comprehensive accurate performance for texture attributes (directivity, crudeness or fineness, etc). Based on Gabor wavelet texture analysis is agreed with human visual system, but its descriptive difference. The pixel position information is lost by color histogram method the different images may have the same color histogram distribution, so that the analysis result to reduce the accuracy. The method of spectral reflection characteristic can distinguish artificiality and natural background but the data obtained by the great influence from outside, in general only suitable for laboratory analysis and battlefield bad adaptability. The above methods achieve the effect of camouflage evaluation respectively from different angles, but the existence of high professional requirements for interpreters, the evaluation of a single, not accurate and evaluation result is not enough visual.

Itti proposed the concept of image saliency[3], the salient characteristics of image analysis results more intuitive but the analysis range is too wide, no specific aim. Followed by a large number of literatures on its improvement and used in various fields and good results have been achieved[6-8]. Most of these methods are only applicable to simple background and are not clear for a complicated background target significant performance. In this paper, according to the optical camouflage principle, firstly the color clustering, color space conversion, DFT conversion, filtering, normalization and a series of processing to the obtained image, get complex background saliency characteristic maps. Using image saliency characteristics on camouflage target and background to carry on the analysis, thus realize the complicated background of optical camouflage effect under the condition of the objective evaluation, by with Itti, HSV color space significance and methods, from the saliency maps look, this paper presents methods more intuitive, reasonable, this method can be used as optical camouflage effect objective evaluation to provide effective means[5].

\section{Image Saliency Analysis}

\subsection{Color cluster}

Optical camouflage stealth technology is based on patterning camouflage. The types of patterning colors are not too much for the military target generally $3 \sim 5$ colors as patterning main color. According to this law, the image clusters by color k-means. K-means clustering is $\mathrm{HCM}$ clustering algorithm and has been applied to various fields. The $\mathrm{n}$ vector $\mathrm{Xi}(\mathrm{I}=1,2, \ldots \ldots, \mathrm{n})$ is divided into $\mathrm{K}$ groups by this algorithm, and ask each group clustering center, makes the non similarity (or distance) value function of index (or objective function) minimum, the clustering center is the color of the class in the smallest and distance between class distance the biggest characteristic, which can effectively reflect the characteristics of the color background advantages, and its advantage is not demanded to know in advance to distribution function of sample to be divided[9].

\subsection{The color space conversion}

Modern research shows that the RGB color space based on the three color synthesis theories suited for color picture tube and other equipment and was more difference with human visual characteristic. In the HSV color space, the luminance component $\mathrm{V}$ didn't relate to color information, the hue $\mathrm{H}$ and saturation $\mathrm{S}$ describe the image color information. The ways of people feel colors are closed connected with HSV. HSV color space reflects the observation color way, which is very suitable for

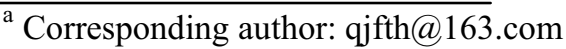


people with visual system to perceive color characteristics of the image processing algorithm [10]. HSV color space is directly corresponded to the human eye color visual characteristics of the three elements. Between channels are independent, the space distance is accorded with human visual characteristics. HSV color space of quantitative results can produce dimension smaller color space, more convenient to describe and control color information, help color features inquires[11] and application. In this paper, the image is converted from RGB color space to HSV color space, in order to content better the analysis and evaluation of optical camouflage effect of the background image.

\subsection{Saliency map generation}

Based on color clustering, the image of HSV color space decomposes by Gaussian pyramid, after using Gaussian template for image processing, gets the Gaussian pyramid structure adjacent two stage to reduce the image size ratio. In order to simulate the human eyes multi-resolution multi-scale sampling and at the same time content the professional requirements for details as high characteristics, the three component and 5 layer of use of Gaussian pyramid model are calculated $H(\sigma), S(\sigma), V(\sigma)$ (where $\sigma \in[1,2, \ldots, 5]$ ). Using the centre-around difference operator produces feature image set, defined $\Theta$ as different scale space characteristics map corresponding point of difference, three component characteristics calculation is as follows:

$$
\begin{gathered}
H(c, s)=|H(c) \Theta H(s)| \\
S(c, s)=|S(c) \Theta S(s)| \\
V(c, s)=|V(c) \Theta V(s)|
\end{gathered}
$$

Where $c \in\{1,2\}, s=c+\delta, \delta \in\{2,3\}$.

Through the above calculation, each characteristic can obtain four center-around the characteristic difference map, and then to the different scale characteristic difference fuse by multi-scale. Because the front of the scale of the Gaussian pyramid structure from 1 to 5 , so that choose in the middle of the scale for a saliency map scale[12], so we can give consideration to the large scale and small scale of local differences and get better effect. Before fusion to the characteristics difference for scale difference, we first need to normalize. In the Itti model defines a normalization operator $N(\cdot)$ that can inhibit differences map of the strong contrast area, and enhance influence power of the having less strong contrast the characteristics difference, in order to burst many saliency characteristic by stacking multiple saliency map $[8,13]$.

The characteristic difference map of normalization does Fourier transform and then nature logarithm spectrum of sin and cosine, and finally to do inverse Fourier transform. Saliency map is normalized above images and linear additive, the characteristic image to merge get hue, saturation and brightness of saliency map respectively. Calculation is as follows:

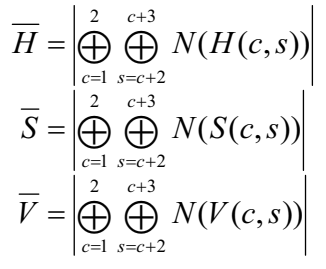

(2)

Where $\oplus$ defined sum operation of characteristic image.

Entropy weight method [14] based on image of each feature weights with information variety, it is more reasonable. Synthesized the saliency of all characteristics and can get saliency map $S$ corresponding to the input image:

$$
\left.S=\omega_{1} N(\bar{H})+\omega_{2} N(\bar{S})+\omega_{3} N(\bar{V})\right)
$$

$\sum_{\text {Where }}^{3} \omega_{n}=1$ value determines the index degrees of importance.

Now, the complicated background area of camouflage target analyses the saliency, as shown in Figure 1:
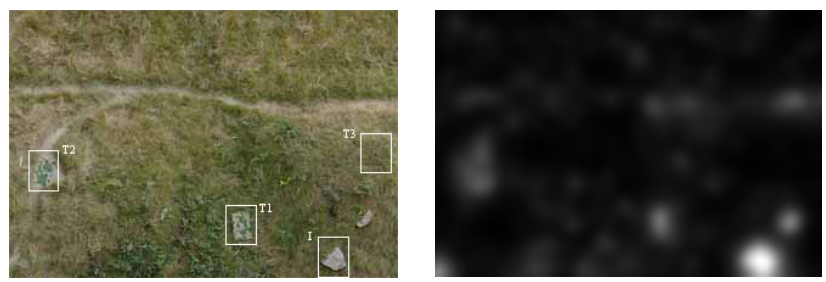

Fig.1 Background image and salience map

In complex background image (Figure 1 left) has three the patterning target, respectively in the image target 1,2 , and 3 . Through the visual observation, we can distinguish road, target 1 , target 2 and interference objects. While the target 3 and background fusion is better, it is difficult to distinguish with visual. Using this algorithm to generate the saliency map, the significance is biggest for interference objects. After the patterning target 1 and 2 significant is a low, but still can easily distinguish out. While target 3 significant is very low, it can't be distinguished in the saliency map (Figure 1 right).

From the above analysis shows that this calculation method can be used for analysis of optical camouflage effect significant in the complicated background conditions, the results reflect the background significant characteristic and the method of camouflage evaluation by the saliency map is feasible.

\section{Simulation and Comparison Algorithm}

In order to verify the validity of this algorithm, the analysis of significant optical patterning effect with different background complexity of the 30 images, and compared with Itti, HSV saliency map method. Results are consistent, choose three images as shown in Figure 2: 


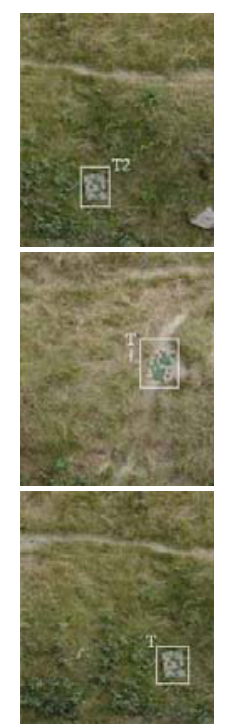

Background image

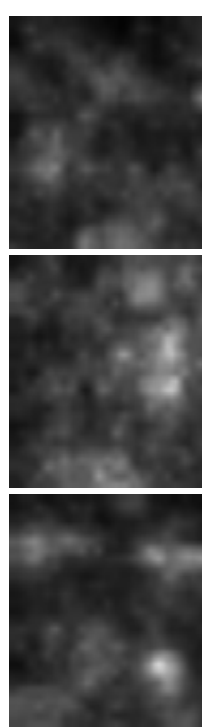

Saliency map of Itti

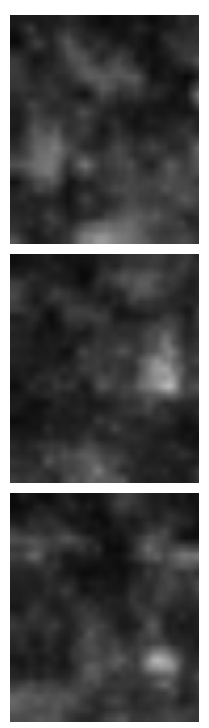

Saliency map of HSV

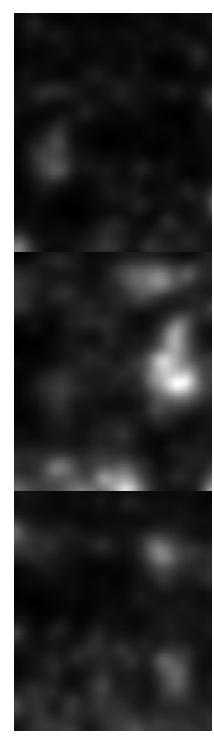

Saliency map of text
Fig.2.The comparison of the salience map

In the background image, not patterning vehicle target 1 , patterning target 2, 3 and 4 , and is easy to cause the background of visual attention road, bridge, bare rock etc.

From Figure 2, the Itti saliency map results from a certain extent reflects the significant features of the original, first discovered the highlighted target 1 and 3, followed by bare rock, target 2 , in the saliency map, while the target 4 is not reflected. The relative Itti saliency map effect, significant area contour of HSV saliency map has been improved, but the difference is not very large. This algorithm the saliency map clearly shows the original of each significant target, such as bare rock, bridge, target 1, 2 and 3, while the target 4 is not reflected in the saliency map due to fuse good with the background.

Although Itti saliency map and HSV saliency map also correctly represent the main significant features of the complicated background, but also shows too much low significant features. It brings heavy interference for the objective evaluation of the complicated background target camouflage effect. This algorithm can effectively filter not significant information mottled in complex background, and after a series of processing makes the saliency map generated more clear, can accurately display the significant matter in the image, consistent with the visual results, convenient interpreter evaluation, non-professionals can also interpret accurately. Therefore, this algorithm can provide the effective means to target camouflage effect evaluation under complex background.

\section{Conclusion}

A significant feature of image can be used to camouflage effect evaluation. This paper aims at the patterning targets in the complex background, get the final saliency map by the corresponding processing, relative to other saliency map generation method, the results obtained from this method is more intuitive, accurate, clear, and can effectively filter out the low significant characteristics of background, extruded the fixation point in the image, consistent with the results and visual. Besides the interference matter, if camouflage target appears in the saliency map, then this camouflage target can easy be found by the reconnaissance equipment, camouflage effect is poor, conversely not easy be found, camouflage effect is good. Thus this algorithm results can objectively evaluate the camouflage effect of target.

A significant characteristics of image to camouflage effect evaluation is convenient, because the background of bare rock and other objects, through the saliency map in the evaluation of camouflage effect was still give the judge to bring some interference, how to effectively eliminate the interference of objects, this is a problem needed to be solved in the next step.

\section{References}

1. Xu WeiDong, Lv Xuliang, Chen Bing. A Model Based on Texture Analysis for the Performance Evaluation of Camouflage Screen Equipment, Acta Armamentarii, 2002, 23(3):229-331

2. Ling Wei, Cheng Yuhua, Gao Hongsheng, Wang Jijun. A Method of Camouflage Evaluation Based on Texture Analysis Model of Gabor Wavelet, Acta Armamentarii, 2007, 28(10):1191-1194

3. Wang Dong, Lv Xuliang, Xu Weidong. Camouflage Application Models with Pixel Frequency Analysis, Journal of PLA University of Science and Technology, 2004, 5(3):74-77

4. Xue Jingjing, Bai Tingzhu. Camouflage Assessment Based on Spectral Reflect Signature, Laser \& Infrared, 2007, 37(10):1078-1084

5. Itti L,Koch C,Niebur E.A model of saliency-based visual attention for rapid scene analysis[J].IEEE Transaction on Pattern Analysis and Machine Intelligence,1998,20(11):1254-1259.

6. Dou Yan, Kong Lingfu, Wang Liufeng. A Computarional Model of Visual Attention Based on Visual Entropy, Acta Optica Sinica, 2009, 29(9):2511-2516

7. Cai Yunxiang, Qin Jianfei, Lv Zhengjian. A Model to Extract the Pattern from Background Based on Visual Attention Model, Optical Technique, 2010, 36(2):302-306

8. Itti L,Koch C. A saliency-based search mechanism for overt and covert shifts of visual attention[J]. Vision Research,2000,40(2000):1489-1506.

9. Bouman C,Sauer K. A generalized Gaussian image model of edge preserving map estimation[J]. IEEE Trans. Image Processing,1993,2(3):296-310.

10. Qin Jianfei, Hu Jianghua, Yang Gaofeng. A New Method to Determine Imitated Pattern Painting Color, Journal of PLA University of Science and Technology, 2011, 12(1):75-78

11. $\mathrm{Xu} \mathrm{Xu,} \mathrm{Zhu} \mathrm{Miaoliang,} \mathrm{Liang} \mathrm{Qianhui,} \mathrm{Waheed}$ Sajjad. A Method of Dominant Colors Eetraction and Representation for CBIR Systems, Journal computer-aided design \& computer graphics, 1999,11(5):385-388

12. Liang Chunying, Wang Guoying, Kang Jinfeng. Image Retrieval Method Based on Salient Regions, Computer Engineering, 2010, 36(5):210-214

13. Ling Yun, Xia Jun, Tu Yan. Detection of Region of 
Interest and its Application in Video Image Quality Assessment, Journal of Southeast University, 2009,39(4):753-757

14. Cheng Qiyue. Structure Entropy Weight Method to Confirm the Weight of Evaluating Index, Systems Engineering-Theory \& Practice, 2010, 30(7):11251128 